\title{
Measuring Burnout in Primary Care Staff
}

Ami Schattner, MD

Hebrew University and Hadassah Medical School, Jerusalem, Israel.

$\mathrm{T}$ o the Editor: Dolan et al. ${ }^{1}$ use a single-item measure to find a $33 \%$ prevalence of burnout (BO) among primary care providers (PCP) and registered nurses. Residents and hospital physicians across all specialties are also often affected by $\mathrm{BO}$ that can adversely influence patient care as well as their own productivity and personal health. ${ }^{2}$ The ability to identify $\mathrm{BO}$ using a single item is certainly important, but the real focus should shift to prevention rather than detection.

Curiosity in the practice of medicine translates into a pleasurable patient-directed learning experience, and each patient seen presents such an opportunity. At the same time, curiosity about the patient's personal aspects acknowledges the patient's unique identity and emotions. This patient-centered attitude is sensed by the patient and family, leading to both patient and physician satisfaction and bonding. ${ }^{3}$ When curiosity is selfdirected, it implies mindfulness and reflection. All these facets of curiosity ensure daily renewal in practice ${ }^{4}$ and may constitute an effective antidote to BO.

Surprisingly, curiosity remains one of the least explored areas in medicine. However, solid evidence already supports the positive long-term effects of interventions promoting mindfulness and reflection on BO of both PCP and hospital physicians, ${ }^{2}$ and highly humanistic clinicians are not only mindful but also curious. ${ }^{5}$ An inherently curious provider may find enjoyment in every patient encounter and may be considerably less susceptible to burnout and depression.

Corresponding Author: Ami Schattner, MD; Hebrew University and Hadassah Medical School, Jerusalem, Israel (e-mail: amischatt@gmail.com).

\section{REFERENCES}

1. Dolan, et al. Using a single item to measure burnout in primary care staff: a psychometric evaluation. Journal of General Internal Medicine. 2015. doi:10.1007/s11606-014-3112-6.

2. Krasner MS, Epstein RM, Beckman H, et al. Association of an educational program in mindful communication with burnout, empathy, and attitudes among primary care physicians. JAMA. 2009;302:1284-1293.

3. Schattner A. Curiosity. Are you curious enough to read on? J R Soc Med. 2015;108:160-164.

4. Hatem CJ. Renewal in the practice of medicine. Patient Educ Couns. 2006;62:299-301.

5. Chou CM, Kellom K, Shea JA. Attitudes and habits of highly humanistic physicians. Acad Med. 2014;89:1252-1258. 